\title{
Correction: Community detection in attributed networks for global transfer market
}

\author{
G. P. Clemente ${ }^{2}$ (1) $\cdot$ A. Cornaro ${ }^{1}$
}

๑) The Author(s), under exclusive licence to Springer Science+Business Media, LLC, part of Springer Nature 2022

\section{Correction to: Annals of Operations Research https://doi.org/10.1007/s10479-021-04439-9}

Author affiliations reversed during proofing and be read as:

Gian Paolo Clemente affiliated to Università Cattolica del Sacro Cuore and Alessandra Cornaro to Università degli Studi di Milano-Biccocca.

Original article has been updated.

Publisher's Note Springer Nature remains neutral with regard to jurisdictional claims in published maps and institutional affiliations.

The original article can be found online at https://doi.org/10.1007/s10479-021-04439-9.

$凶$ A. Cornaro

alessandra.cornaro@unimib.it

G. P. Clemente

gianpaolo.clemente@unicatt.it

1 Department of Statistics and Quantitative Methods, University of Milano - Bicocca, Via Bicocca degli Arcimboldi 8, 20126 Milano, Italy

2 Dipartimento di Matematica per le Scienze Economiche, Finanziarie e Attuariali, Universitá Cattolica del Sacro Cuore, Largo Gemelli, 1, 20123, Milano, Italy 